\title{
G protein signaling of native somatostatin receptors 2 and 5 in pituitary cells using a fluorescence-based membrane potential assay
}

\author{
Thomas Günther and Stefan Schulz
}

\author{
Jena University Hospital - Friedrich Schiller University Jena
}

Objectives:

Somatostatin and dopamine receptors are the major $\mathrm{G}_{\mathrm{i}}$-coupled receptors in somatotrope cells that inhibit hormone secretion from the anterior pituitary. Somatostatin analogues (SSA) are successfully used in the treatment of neuroendocrine disorders, such as neuroendocrine tumors (NETs). Octreotide and Pasireotide exert their effects on multiple Somatostatin receptor subtypes, mainly sst2 and sst5. Dopamine receptor 2 (D2R) is expressed in various tumors, among them NETs. The chimeric compound Dopastatin (BIM23A760) was designed to address both, sst2 and D2R. Here we adapted a novel fluorescence-based screening assay to characterize somatostatin and dopamine receptor signaling in a timeresolved manner. This minimal-invasive technique provides a robust and reliable read out for ligand-induced receptor activation in permanent cell lines and primary pituitary culture.

Methods:

In this study we evaluate the suitability of a fluorescent imaging plate reader (FLIPR) assay for the characterization of SSA for permanent cell lines and primary pituitary culture expressing endogenous somatostatin receptors. The mouse pituitary cell line AtT-20 expresses two somatostatin receptor subtypes (sst2/5) and $G$ protein-coupled inwardly rectifying potassium channels (GIRK1/2). These channels create the molecular basis to generate the assay read out. Receptor expression of AtT-20 cells was confirmed by RT-PCR and subtype specific antibodies. Cells loaded with a proprietary voltage-sensitive dye were exposed to various somatostatin-receptor subtype specific compounds. Agonistinduced decrease of fluorescence signal was detected.

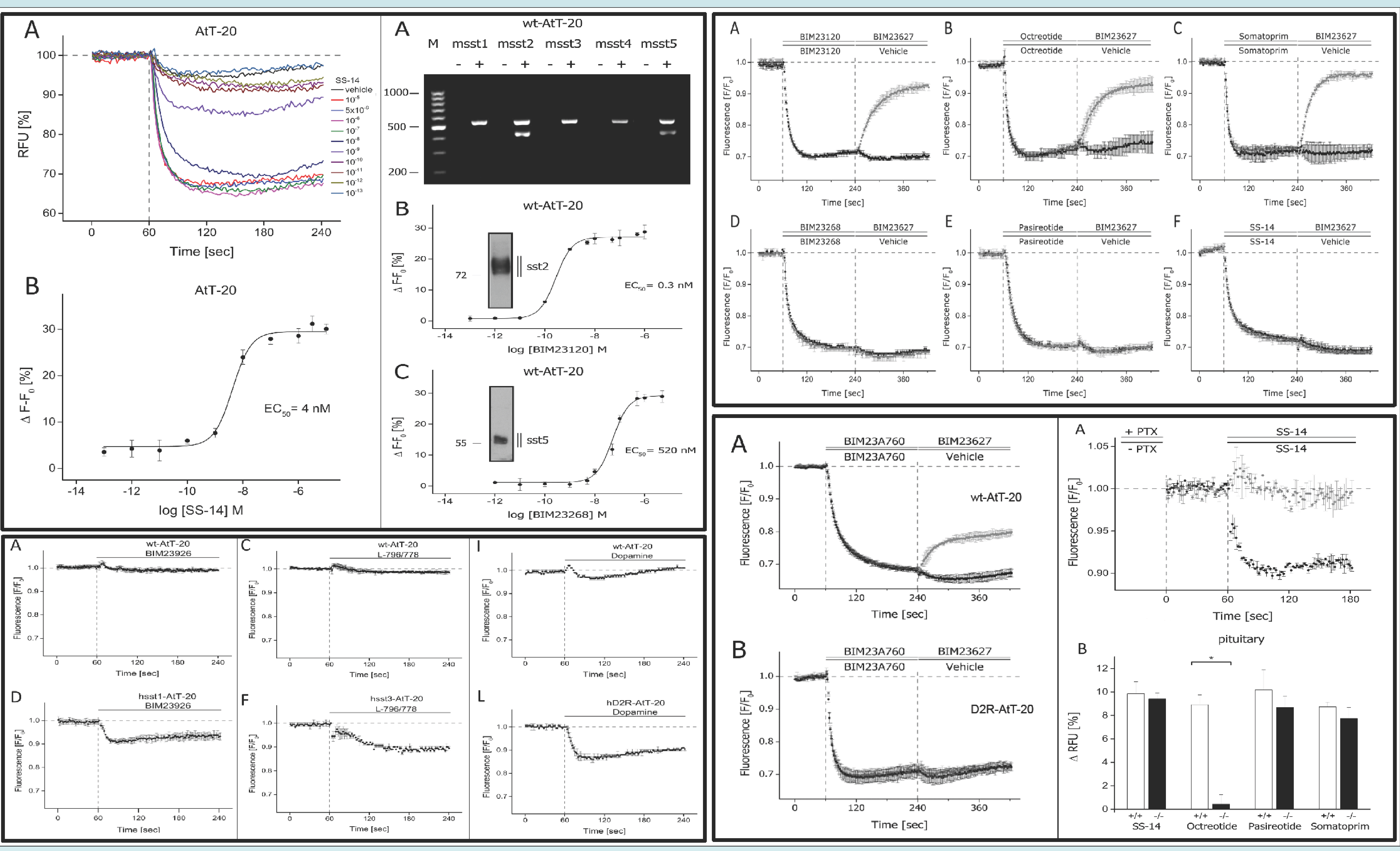

Results:

Among the compounds tested, only substances binding to sst2/5 induced a decrease of fluorescence signal. A pharmacological characterization of multi-ligand SSA was undertaken with subtype-specific antagonists for sst2 (BIM23627). Pasireotide displays its effect predominantly on sst5 even in the presence of sst2, while Octreotide acts as sst2 agonist. The effects of BIM23A760 (Dopastatin) can be partially blocked (in a $1: 1$ molar ratio of agonist : antagonist) with BIM23627 in wt-AtT-20 cells, but not in the presence of D2R. This approach was used to proof accordance of in vitro activity and receptor binding data that are usually obtained by membrane isolates. In contrast, exposure to BIM23926 (sst1-selective), L-796/778 (sst3-selective) or L-803/087 (sst4-selective) did not produce any change in fluorescent signal intensity. However, after heterologous expression of sst1, sst3 or sst4 receptors BIM23926 and L-796/778 but not L-803/087 promoted a reduction in fluorescent signal indicating that sst1 and sst3 receptors can also couple to GIRK channels.

Conclusions:

This fluorescence-based method is an efficient and robust tool in the pharmacological characterization of G Protein-coupled receptors.

A fast and reliable in vitro screening of novel SSA on a medium scale can be performed with this ion channel assay. Also sst1- and sst3-active compounds can be further evaluated. Dopastatin is found to display intrinsic activity at sst2 and D2R.

References:

Huang R, Griffin SA, Taylor M, Vangveravong S, Mach RH, Dillon GH, Luedtke RR (2013) The effect of SV 293, a D2 dopamine receptor-selective antagonist, on D2 receptor-mediated GIRK channel activation and adenylyl cyclase inhibition. Pharmacology 92: 84-89

Knapman A, Connor M (2015) Fluorescence-based, high-throughput assays for mu-opioid receptor activation using a membrane potential-sensitive dye. Methods in molecular biology (Clifton, NJ) 1230: 177-185

Saveanu A, Gunz G, Guillen S, Dufour H, Culler MD, Jaquet P (2006) Somatostatin and dopamine-somatostatin multiple ligands directed towards somatostatin and dopamine receptors in pituitary adenomas. Neuroendocrinology 83: 258-263

Walsh KB (2011) Targeting GIRK Channels for the Development of New Therapeutic Agents. Frontiers in pharmacology 2: 64

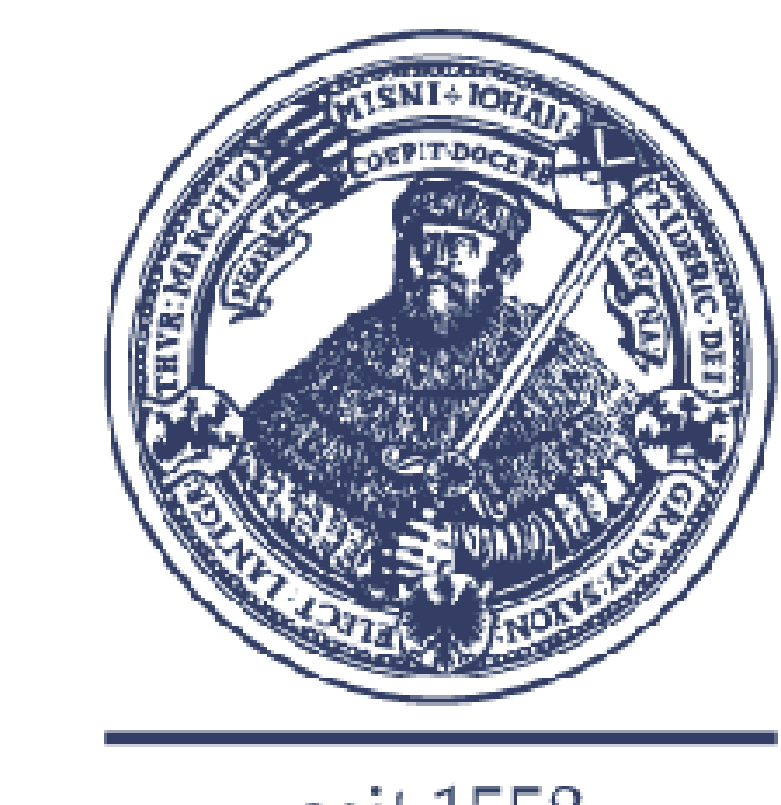

